\title{
El Caso Victor: dificultades metacognitivas en la resolución de problema* $^{*}$
}

\section{Case Study "Victor": metacognitive difficulties in problem solving}

\author{
Tânia Cristina R. S. Gusmão** \\ José Antonio Cajaraville*** \\ Vicenç Font ${ }^{* * * *}$ \\ Juan D. Godino ${ }^{* * * * *}$
}

\begin{abstract}
Resumen
Es posible una mejor comprensión de las prácticas matemáticas realizadas en el proceso de resolución de problemas si se toma, como unidad mínima de análisis, configuraciones cognitivas e metacognitivas. Sin embargo, en este artículo, se presenta con profundidad la parte metacognitiva de un modelo de análisis. Concretamente, se pretende, bajo un examen cuidadoso de un estudio de caso, teniendo como sujeto un alumno del tercer curso de la etapa de Enseñanza Secundaria Obligatoria del Estado Español que contestó a una Prueba de Habilidades Metacognitivas, demonstrar el papel que juegan los procesos metacognitivos para explicar las dificultades de estudiantes en la actividad de resolución de problemas. Se concluye que las dificultades de estudiantes pueden estar relacionadas tanto con sus carencias cognitivas como metacognitivas.
\end{abstract}

Palabras-Clave: Cognición. Metacognición. Resolución de Problemas. Educación Matemática.

\begin{abstract}
A better understanding of the mathematical practices performed in the process of problem solving is possible, when taken into consideration - as the minimum unit of analysis - both cognitive and metacognitive configurations. In this paper, the metacognitive aspect of an analysis model is presented in-depth. The goal, here, is demonstrating the role played by metacognitive processes to explain difficulties students have in solving problems. In order to do so, a case study involving a third grade student from Spain's high-school educational

\footnotetext{
* Este artículo es una versión ampliada, en términos teóricos y metodológicos, de la comunicación, intitulada Modelo de análise do conhecimento cognitivo e metacognitivo, presentada en el XIII Ciaem, 2011, RecifeBrasil.

** Doctora en Didáctica de las Matemáticas por la Universidad de Santiago de Compostela (USC). Profesora Titular en la Universidade Estadual do Sudoeste da Bahia (UESB), Vitória da Conquista, Bahia, Brasil. Dirección Postal: Av. Braulino Santos, 1125, Edifício Versalhes apt ${ }^{\circ} 303$, Candeias, CEP: 45028-170,Vitória da Conquista, Bahia, Brasil. E-mail: professorataniagusmao@gmail.com.

**** Doctor en Didáctica de las Matemáticas por la Universidad de Santiago de Compostela (USC). Profesor Titular en la Universidad de Santiago de Compostela, Santiago de Compostela, Coruña, España. Dirección Postal: Avenida Xoán XXIII s/n, Campus Norte, 1578, Santiago de Compostela, A Coruña, España. E-mail: ja.cajaraville@usc.es.

Doctor en Filosofía y Ciencia de la Educación por la Universidad de Barcelona (UB). Profesor Titular en la Universidad de Barcelona, Barcelona, España. Dirección Postal: c/ Figols, 15. 08028, Barcelona, España. Dirección Postal: Avenida Xoán XXIII s/n, Campus Norte, 1578, Santiago de Compostela, A Coruña, España. Email: vicencfont@ono.com.

${ }_{* * * * *}$ Doctor en Matemáticas por la Universidad de Granada (UG). Profesor Catedrático en la Universidad de Granada, Granada, España. Dirección Postal: Facultad de Educación, Campus de Cartuja, 18071, Granada, España.E-mail: jgodino@ugr.es.
} 
system is carefully examined. The subject answered a Metacognitive Skills Test, and, based on the analysis, the conclusion is that the difficulties students have might be related to either their cognitive or their metacognitive needs.

Keywords: Cognition. Metacognition. Problem Solving. Mathematics Education.

\section{Introducción}

Recientemente, en diferentes países, se han propuesto reformas curriculares que asumen que la Resolución de Problemas (RP) es la actividad fundamental en la construcción del conocimiento matemático de los alumnos (LESH; ZAWOJEWSKI, 2007). Para citar solo dos ejemplos, (1) tenemos que la propuesta de estándares y principios del NCTM (2003) contempla como uno de los cinco estándares de procesos del pensamiento matemático la RP y (2) que los currículums por competencias que se están elaborando actualmente, influenciados por el estudio Pisa (OCDE, 2003), contemplan, como una de las principales, la competencia en la RP.

La investigación sobre la RP está estrechamente relacionada con este interés en incorporar la resolución de problemas en el currículum. Si bien hay muchas líneas diferentes en la investigación sobre la RP, una de las más productivas es la que se ha interesado en responder a la pregunta ¿Cuál es el pensamiento que activan y manifiestan los alumnos cuando resuelven problemas? Schoenfeld (2007) sugiere que las dimensiones - conocimiento o recursos básicos de matemáticas; estrategias cognitivas o heurísticas para representar y explorar los problemas; estrategias metacognitivas acerca del funcionamiento cognitivo propio del individuo; e las creencias, las actitudes y componentes afectivos en la concepción del individuo acerca de las matemáticas y la resolución de problemas - pueden explicar el éxito o fracaso de los estudiantes, pero no explican cómo y por qué los estudiantes exhiben esos comportamientos al resolver problemas.

Durante las décadas 80 y 90 del siglo pasado se realizaran muchas investigaciones sobre el papel que jugaba la metacognición en la actividad matemática de los alumnos, consiguiéndose un cierto consenso sobre la importancia de la metacognición para el pensamiento matemático efectivo y la RP (CAJ, 1994; CLARKE; STEPHENS; WAYWOOD, 1992; FERNANDES, 1988; GAROFALO; LESTER, 1985; GONZÁLEZ, 1997; GOOS, 1994; LESTER, 1994; LESTER; GAROFALO, 1982; SCHOENFELD, 1985, 1992; SILVER; MARSHALL, 1990; YUSSEN, 1985). De hecho, hay investigadores que afirman que una de las causas fundamentales de las dificultades en la RP consiste en una falta de habilidad de los 
alumnos para monitorizar y regular activamente sus procesos cognitivos (GAROFALO; LESTER, 1985; LESTER; GAROFALO, 1982; SCHOENFELD, 1992; KAUNE, 2006).

A partir de la década de los 90, la investigación sobre la RP dejó de limitarse al estudio de aspectos cognitivos y metacognitivos, ampliándose al estudio de aspectos socioculturales y semióticos. Por otra parte, algunos de los programas de investigación que se están desarrollando actualmente, en el área de la Educación Matemática, se plantean el estudio de la metacognición, y, más en general, la RP, desde el marco teórico que dichos programas ofrecen; este es el caso de las investigaciones de Rodríguez (2005) y Rodríguez, Bosch y Gascón (2008), que utilizan el enfoque antropológico de lo didáctico (CHEVALLARD, 1992) para mostrar cómo puede integrarse la RP en el proceso de enseñanza-aprendizaje de las matemáticas por medio de los denominados Recorridos de Estudio e Investigación, o el caso de las investigaciones de Gusmão (2006) y Gusmão, Font y Cajaraville (2009) en las que se reflexiona sobre la metacognición, utilizando el enfoque ontosemiótico de la cognición e instrucción matemática (GODINO; BATANERO; FONT, 2007).

El trabajo que aquí se presenta está interesado en investigar el papel de la metacognición en las prácticas de RP, para ello se considera que, para el análisis de las prácticas matemáticas realizadas en el proceso de resolución de problemas, es necesario considerar como unidad mínima una herramienta compuesta por una configuración cognitiva y una configuración metacognitiva. Sin embargo, en este artículo, se presenta con detalle solamente la parte metacognitiva. Con respecto a la configuración cognitiva, vale resaltar, de acuerdo con Font, Planas y Godino (2010) que para la realización de una práctica matemática y para la interpretación de sus resultados como satisfactorios se necesita poner en funcionamiento determinados conocimientos (situaciones-problema, lenguaje, conceptos, proposiciones, procedimientos y argumentos) los cuales se articulan formando una configuración cognitiva (parte izquierda de la Figura 2, adaptada de Font y Godino, 2006, p. 69):

Si consideramos los componentes del conocimiento que es necesario que el agente tenga para la realización y evaluación de la práctica que permite resolver una situación problema (e.g., primero plantear y después resolver un sistema de dos ecuaciones con dos incógnitas) vemos que ha de utilizar un determinado lenguaje verbal (e.g, solución, ecuación) y simbólico (e.g., x, =). Este lenguaje es la parte ostensiva de una serie de conceptos (e.g., ecuación, solución), proposiciones (e.g., si se suma el mismo término a los dos miembros de la ecuación se obtiene una ecuación equivalente) y procedimientos (e.g., resolución por sustitución, por igualación) a utilizar en la elaboración de argumentos para decidir si las acciones simples que componen la práctica, y ella misma entendida como acción compuesta, son satisfactorias. Consideramos que cuando un agente realiza y evalúa una práctica matemática tiene que activar un conglomerado formado por algunos de los objetos citados anteriormente (o todos): situaciones-problema, lenguaje, conceptos, 
proposiciones, procedimientos y argumentos. (FONT; PLANAS; GODINO, 2010, p. 95-96).

Con respecto a las prácticas matemáticas, estas serán consideradas, como la unión de una práctica actuativa mediante la que se realiza la lectura y producción de textos matemáticos y de una práctica discursiva (de reflexión sobre la práctica actuativa) que pueden ser reconocidas como matemáticas por un interlocutor experto. El sentido de las prácticas matemáticas, de acuerdo con Godino y Batanero (1994), viene determinado por la función que esta práctica desempeña en los procesos de RP, o bien para comunicar a otro la solución, validar la solución y generalizarla a otros contextos y problemas.

\section{Marco teórico}

De la revisión de la literatura en torno al referente metacognición podemos observar que el panorama de constructos teóricos elaborados en torno a este referente, traza dos grandes divisiones de contenido con respecto al concepto de metacognición: se concibe como producto y como proceso de la cognición. Y observamos que de esas divisiones van surgiendo varias ramas o subdivisiones. En el organigrama que sigue (Figura 1), Gusmão (2006) intenta representar como están organizados los constructos básicos o más generales, o al menos los más mencionados por los teóricos e investigadores, que van diseñando dicho referente.

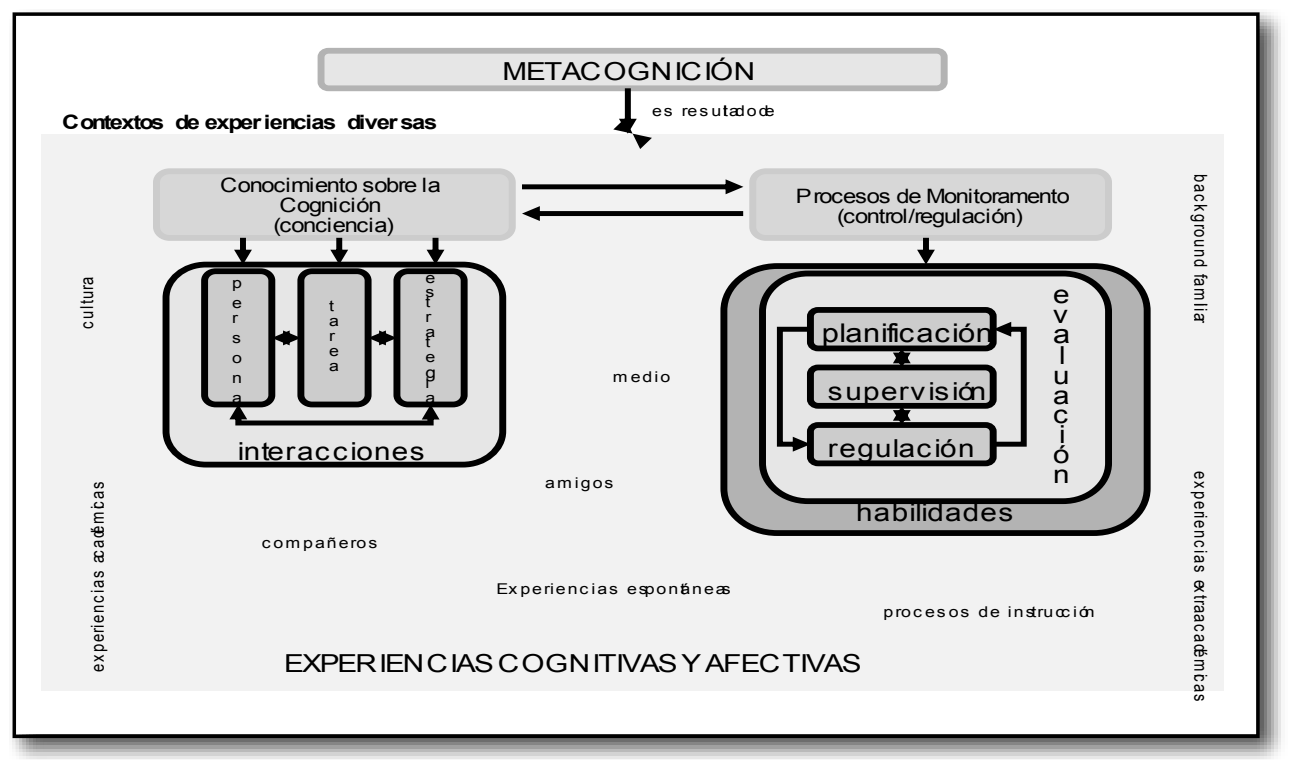

Figura 1 - Organigrama: La metacognición y sus principales componentes Fuente: Gusmão (2006, p. 90)

La interacción entre estas dos grandes componentes (divisiones) puede darse de la siguiente forma: por una parte, el desarrollo del conocimiento metacognitivo sobre las 
variables que lo componen permite que la actividad de monitoreo ocurra con más eficacia sobre los emprendimientos cognitivos; si se recurre a una actividad de monitoreo, como por ejemplo, una planificación cuidada, con el fin de incrementar, pongamos, por caso, el dominio del conocimiento de un tema (y por tanto aumentar el conocimiento metacognitivo sobre la tarea), habrá una influencia del conocimiento metacognitivo sobre el proceso de regulación. Por otra parte, al evaluar la eficacia de una estrategia (cognitiva o metacognitiva) y su potencial utilización en otras situaciones (actividad reguladora), puede ocurrir un aumento del conocimiento metacognitivo sobre la variable estrategia. De modo que hay, constantemente, una interacción entre ambas componentes (GONÇALVES, 1996).

Observamos que tanto el conocimiento acerca de la cognición, como las actividades de monitoreo emergen (y acompañan) las experiencias que uno va adquiriendo con las tareas que maneja, con las personas de su alrededor, con el uso de determinadas estrategias, es decir, con las experiencias que según Flavell $(1976,1981,1987)$ y Flavell y Wellman (1977) están relacionadas con el progreso hacia las metas. Destacamos, aún, las experiencias que los sujetos adquirieron (y van adquiriendo) en diversos contextos, nombrados de muchas formas: background familiar, la cultura, los amigos, la calle y en el ámbito extra académico de forma general. También, las vertientes de la metacognición guardan relación con el factor edad y con los significados que uno atribuye al mundo de objetos a su alrededor. De aquí, podemos inferir que la metacognición tiene sus raíces en los hechos, en el uso, y es, ante todo, un constructo que emerge de la práctica, que representa una porción de las experiencias y del conocimiento que uno adquiere del mundo, con el mundo y en el mundo.

\subsection{Relación entre metacognición y prácticas de RP}

En las discusiones sobre la metacognición en tanto que conocimiento sobre la cognición, se incluyen tres tipos de conocimiento que han recibido las etiquetas de conocimiento declarativo (conocimiento proposicional que se refiere al saber qué acciones pueden emprenderse para llevar a cabo una tarea y qué factores influyen en el rendimiento, o sea, incluye conocimientos sobre sí mismo como aprendiz y sobre factores que influencian su actuación), conocimiento procedimental (se refiere al saber cómo aplicar dichas acciones y muchos de esos conocimientos son conocidos como heurísticos y estrategias) y conocimiento condicional (se refiere al saber por qué, usamos el conocimiento declarativo y el procedimental. Es un conocimiento importante, porque ayuda a actuar con más eficacia en la 
selección y uso de estrategias). (SCHRAW 2001; MATEOS, 2001; CARREL; GAJDUSEK; WISE, 2001, entre otros). Estos tres aspectos, se trata, en nuestra opinión, de una clasificación que en ciertos casos resulta difícil de aplicar, sobre todo cuando se intenta aplicar a la resolución de problemas.

Dada esta dificultad, y puesto que nuestro interés central reside en comprender las prácticas matemáticas de los alumnos en el proceso de RP, hemos optado por hacer una revisión de esta clasificación desde una perspectiva pragmatista. De acuerdo con esta perspectiva nos interesa no limitarnos únicamente a explicaciones mentalistas, puesto que consideramos que el hombre no es un ser que sólo piensa, comprende... Sino que también es un ser que actúa, toma decisiones etc.

Las prácticas matemáticas que pone en funcionamiento el saber qué, cómo, por qué y, también, cuándo, son indicadores de que fue activado un estado de conciencia, y, en nuestra opinión, también pueden funcionar, en algunos casos, como indicadores de estados de conciencia que se pueden considerar como metacognitivos.

Puesto que el conocimiento acerca de los propios procesos y productos cognitivos llevado a cabo por una persona (cognitiva y afectiva) sufre influencias de contextos sociales diversos (familia, escuela, procesos de instrucción estándar y no-estándar...), que juntos construyen una historia de vida de un sujeto, nos inclinamos por entender la metacognición como un conocimiento teórico-práctico-social, que acompaña a la cognición (interaccionando ambos continuamente, sin que se pueda considerar que uno determina al otro de manera mecánica), pudiendo ser desarrollado y/o incrementado al mismo tiempo que el conocimiento cognitivo es desarrollado, y como tal es resultado de las exigencias de la conducta social efectiva y satisfactoria y que además, se usa y se cambia bajo constricciones contextuales.

La metacognición suele manifestarse desde estados (accesos) de conciencia automática hasta estados de conciencia deliberada (control deliberado), desde estados pasivos a estados activos de conciencia. En el intervalo entre un estado pasivo y uno activo podemos pensar en muchos niveles (estados) de conciencia, desde el más vago al más elevado, y por tanto, podemos pensar en muchos niveles de metacognición. Es en este sentido que presentamos una configuración metacognitiva que, actuando junto con la configuración cognitiva (propuesta por el EOS), en tanto que herramientas de análisis, puedan contribuir a una mejor comprensión de las prácticas que realizan los estudiantes para resolver problemas, y, por tanto, para una mejor comprensión de las conductas matemáticas de éstos. 
Para la configuración metacognitiva que proponemos a continuación, consideramos tres niveles que, a su vez, pueden ser desglosados en otros más específicos, si es necesario. Es importante señalar que a pesar de esta separación, dichos niveles, en el proceso de RP, actúan de manera conjunta, retroalimentándose continuamente.

\subsection{Configuración metacognitiva}

Para la realización de la práctica, como por ejemplo resolver un problema que le suponga un grado de dificultad importante, un resolutor experto pondrá en funcionamiento una configuración epistémica/cognitiva (según se mire desde la perspectiva institucional o personal), pero para ello tiene que tomar una serie de decisiones de gestión de los componentes de la configuración epistémica a lo largo del proceso de resolución (coordinación, planificación/organización, supervisión/control, regulación y revisión/evaluación que pueden ser automáticas o declaradas en función del tiempo, instrumentos disponibles etc.). Para el caso particular de las prácticas que realizaron los alumnos en el contexto de nuestras tareas, propondremos, conforme Gusmão (2006), una reconstrucción hipotética de una configuración metacognitiva adaptada a cada situación problema, que de modo general la podemos describir de la forma indicada en el Cuadro 1: 


\section{Gestiones primarias (metacognición primaria) \\ I-Comprender el enunciado \\ II- Tomar conciencia de las exigencias y condiciones del problema. \\ II-Decidir o elegir los pasos que supuestamente levará a la solución.}

Consideraciones para una Configuración Metacognitiva Institucional de Referencia

Se es un experto en la materia, las decisiones serán rápidas (e incluso, en algunos casos, automáticas) y las argumentaciones sobre la bondad del plan adoptado serán precisas y de acuerdo con los conocimientos institucionales.

Las gestiones tienen un nivel relativamente semiautomático de procesos de supervisión, regulación y evaluación. De modo general, las acciones metacognitivas iniciales que se esperan para este nivel serán, sobre todo, de comprensión y de organización/planificación.

\section{Gestiones secundarias (metacognición secundaria)}

Cuando no se trata de gestiones rápidas o automáticas debido a la complejidad del problema, serán necesarios periodos de espera y de nuevos planteamientos. Todo eso, implican gestiones deliberadas de supervisión, regulación y evaluación más reflexivas que las que se dan en la primera.

I- Acción supervisiva- el resolutor, implícita o explícitamente, hace cuestionamientos del tipo ¿estoy siguiendo correctamente el plan previsto? Tal supervisión le conduce (y garantiza) a un mayor rendimiento.

II- Acción regulativa- el resolutor implícitamente o explícitamente hace cuestionamientos del tipo si no consigo los objetivos o no cumplo las condiciones impuestas, qué puedo corregir o qué nuevo camino puedo emprender. Se da cuenta de que se equivocó y sobre todo se pregunta cuándo o dónde se equivocó.

III- Acción evaluativa/verificativa- el resolutor explícitamente hace cuestionamientos del tipo ¿Ação Evaluativa/verificativa- “¿estoy respondiendo correctamente a la tarea?” ¿La solución que doy es la que resuelve el problema? Estos tipos de preguntas son indicios de la existencia consciente de un proceso de evaluación/verificación final de las acciones emprendidas.

$$
\text { Gestiones para una metacognición ideal }
$$

Lo que caracteriza este tercer nivel metacognitivo es el recurso deliberado de procesos cognitivos de características muy generales (pensamiento metafórico, analógico, particularización, generalización, transferencia, contextualización, descontextualización, cambio de representación, resolución alternativa, una solución original, etc.), los cuales se proponen como nuevas alternativas (mucho más conscientes y reflexivas) a las demandas de supervisión, regulación y evaluación anteriores.

\section{Cuadro 1 - Configuración Metacognitiva}

Fuente: Gusmão (2006, p. 105)

Aunque hemos puesto los tres niveles de metacognición separados uno del otro, hay que pensarlos como un proceso continuo que se desarrolla en espiral. En muchos casos será suficiente el nivel primario de metacognición (cuando, por ejemplo, un resolutor experto se enfrenta a un problema que para él es simple). Sólo aparecerán explícitamente los niveles secundario y terciario cuando el resolutor se enfrente a una situación problema cuya complejidad le obligue a ponerla en funcionamiento.

La configuración metacognitiva institucional (de un resolutor ideal), será tomada como referencia para evaluar las configuraciones metacognitivas personales de los estudiantes. En la confección de esta configuración epistémica (o de referencia) se han tenido en cuenta los 
siguientes aspectos: 1) la actividad metacognitiva de los profesores de matemáticas que han resuelto el conjunto de nuestros problemas que posteriormente se propusieron a los alumnos, 2) la revisión de la literatura sobre la metacognición que hemos consultado y 3) la experiencia de la doctoranda y de los directores de tesis en la resolución de problemas en la formación continua de los profesores y 4) la opinión de expertos de la metacognición que hemos consultado. Posteriormente, esta configuración se ha refinado teniendo en cuenta matices sugeridos por las respuestas de algunos estudiantes. En la Figura 2 presentamos un esquema de análisis de las prácticas de RP:

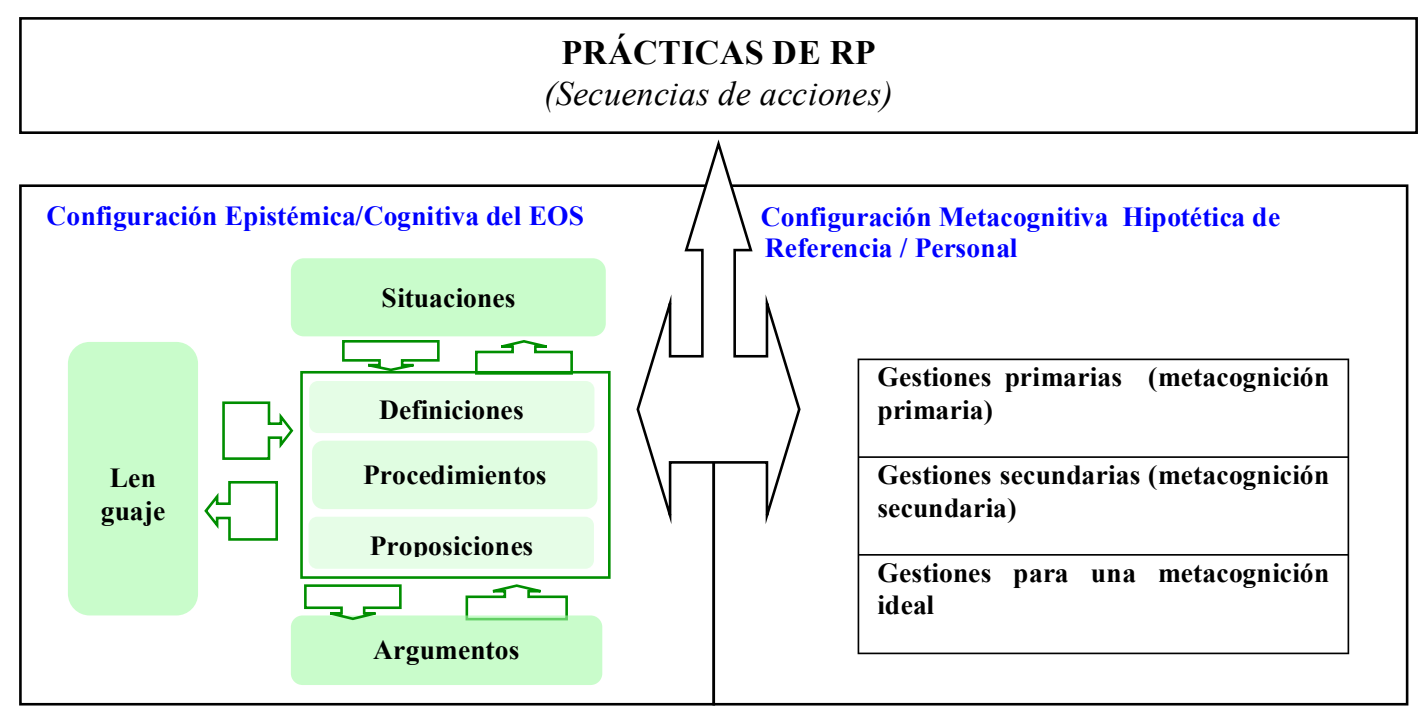

Figura 2 - Herramientas cognitivas y metacognitivas Fuente: Gusmão (2006, p. 106)

Con este esquema queremos representar que si bien, por una parte conviene, para el análisis de las prácticas de RP, considerar por separado los constructos configuración cognitiva y metacognitiva, que a su vez están descompuestas en sus elementos constitutivos, queremos señalar que vemos estos constructos formando parte de un todo integrado, que en su conjunto contribuye a explicar la realización de dicha práctica.

\section{Material e método}

Uno de los objetivos de la didáctica de las matemáticas es comprender los procesos de enseñanza-aprendizaje de las matemáticas, lo cual exige investigaciones de tipo teórico que permitan la creación y el desarrollo de marcos teóricos que puedan ser aplicados a dichos procesos. Como nuestro objetivo general es comprender mejor las prácticas realizadas por los sujetos al resolver problemas, nos hemos planteado una investigación que desarrolle 
herramientas teóricas que se puedan aplicar al análisis de la RP (fase teórica) y las hemos aplicado a una muestra de alumnos (fase empírica).

La fase teórica ha consistido, básicamente, en un análisis de fuentes documentales de tipo epistemológico, cognitivo y didáctico, adoptando una posición propia sobre las diferentes fuentes, lo cual se ha ido produciendo de manera dialéctica con el avance de la fase empírica de la investigación.

La fase empírica de la investigación que se presenta se ha focalizado, sobre todo, en cuestiones cognitivas/metacognitivas (significados personales de los alumnos). La finalidad ha sido, sobre todo, descriptiva. Se ha tratado de una investigación exploratoria ya que no se pretende generalizar a otros contextos o poblaciones y el nivel de análisis es puntual ya que se investiga cuestiones ligadas al estudio de una cuestión matemática específica en un contexto determinado.

\subsection{Muestra}

Se diseño una Prueba de Habilidades Metacognitivas (PHM), que fue contestada por 185 alumnos que cursaban el tercer curso de la etapa de Enseñanza Secundaria Obligatoria (ESO) del Estado Español. Para este artículo, presentamos la respuesta de un alumno.

\subsection{Cuestionario-prueba de habilidades metacognitivas}

Para evaluar las habilidades metacognitivas de los alumnos, fue construido un cuestionario llamado Prueba de Habilidades Metacognitivas (PHM) tomando como punto de partida la prueba inicial de habilidades de aprendizaje ${ }^{1}$ del Instituto de Evaluación y Asesoramiento Educativo (IDEA), que adaptamos para el nivel de $3^{\circ}$ de ESO.

Hay algunas características de la PHM que queremos resaltar. Sobre el instrumento, este no se basa en automatismos. Está compuesto por un conjunto de problemas no-rutinarios, lo que significa que el estudiante no dispone (en nuestra opinión) de habilidades estandarizadas para resolverlo, aunque podría tener recursos adecuados para intentarlo.

Los problemas de la PHM fuerzan al resolutor a tomar decisiones, hacer elecciones y enjuiciamientos sobre si lo que se plantea permite o requiere la utilización de modelos

\footnotetext{
${ }^{1}$ Autoría de la Dra. Amparo Moreno. Departamento de Psicología Evolutiva y de la Educación-Universidad Autónoma de Madrid.
} 
conocidos o hay que emprender nuevos caminos. En la medida del posible, intentan que el alumno se exprese libremente, huyendo de respuestas estereotipadas.

Los problemas no-rutinarios de la PHM rompen con los tipos de estrategias (algorítmicas, cálculo rutinario) habituales, produciendo un desequilibrio (en el sentido de Piaget) que requiere pensamientos y acciones conscientes.

Realizamos un primer experimento, a través de un estudio piloto, con la PHM que contenía 12 problemas. El estudio piloto tuvo un carácter exploratorio, o sea, tuvo la finalidad de practicar e intentar prever algunas dificultades de aplicación. Una vez corregidas las respuestas y discutidos los resultados, realizamos una serie de modificaciones en algunas preguntas que dieron lugar a un nuevo instrumento aplicado, en dos etapas.

El nuevo instrumento, ahora con 15 problemas, quedó dividido en dos partes interrelacionadas. Una primera (parte principal), conteniendo nueve problemas que intentamos vaciar al máximo del formalismo simbólico propio de la matemática. Una segunda (parte complementaria), aplicada una semana después de la primera, conteniendo seis problemas, donde se introducen signos algebraicos (con carácter comunicativo), se reformulan tres de los problemas anteriores y se presenta uno nuevo que permite, aunque no exige, el uso de fórmulas de áreas de figuras simples (correspondientes a Educación Primaria).

Las cuestiones implicadas en esta prueba requieren del resolutor la capacidad de clasificar, ordenar, seriar, combinar, descomponer, aproximar, hacer suposiciones, simplificar, generalizar, entre otras. Son cuestiones que implican la liberación del pensamiento en relación con los objetos.

Además, la PHM es un instrumento de naturaleza abierta, más subjetiva que objetiva (a pesar de existir algunas cuestiones de múltiple elección el alumno debe siempre justificar su elección o decisión), cognitiva y metacognitiva y más o menos independiente del currículo oficial del curso. La descripción completa de la PHM se encuentra en Gusmão (2006).

\subsection{Entrevistas}

Por último se seleccionaron algunos alumnos que fueron entrevistados para profundizar sobre sus respuestas a la PHM. Las entrevistas se realizaron en un plazo muy corto: 20 minutos con cada estudiante, ya que tuvimos que aprovechar momentos del propio horario normal de las clases de matemáticas para estar con ellos, contando, siempre, con el 
permiso de su profesor. Optamos por la entrevista semiestructurada, toda vez que una de sus ventajas es de permitir la captación inmediata y corriente de la información deseada. Es un tipo de entrevista flexible y admite adaptaciones cuando son necesarias (LUDKE; ANDRÉ, 1986).

\section{El caso Víctor}

Víctor es un estudiante de $3^{\circ}$ de E.S.O. (15 años) que, además de haber contestado a la PHM, nos concedió una entrevista para aclaraciones sobre dicha prueba. De su entrevista, recortamos las aclaraciones dadas por él al problema de las 9 bolitas. El análisis de este caso sigue en dos momentos (un protocolo escrito y otro oral), trazando, inicialmente, las configuraciones cognitivas y metacognitivas institucionales que sirvieran de referencia para evaluar la tipología de configuraciones cognitivas y metacognitivas personales de Víctor. Sin embargo, en este artigo, se profundizará el análisis de la configuración metacognitiva.

Primer momento: el protocolo escrito (Figura 3).

\section{Problema de las 9 bolitas}

Fíjate cómo pensó Rocío la forma de averiguar cuál era la bolita más ligera, efectuando una única pesada:

Cogió dos cualquiera de las bolitas y puso una en cada plato:

a) Si una pesaba menos, esa sería la más ligera;

b) Si pesaban lo mismo, la que quedó sin pesar sería la más ligera.

Ahora tienes nueve bolitas semejantes, también una de ellas más ligera que las otras. ¿Cómo podrías descubrir cuál es, en dos pesadas?

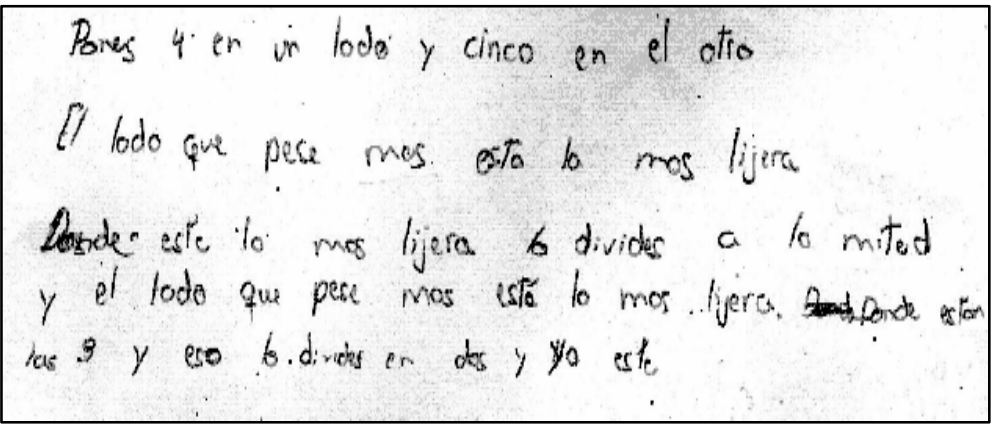

Pones 4 en un lado y cinco en el otro.

El lado que pese más está la más ligera.

Donde está la más ligera lo divides a la mitad y el lado que pese más está la más ligera. Donde están las 3 y eso lo divide en dos y ya está.

Figura 3 - Protocolo escrito de Víctor

Fuente: Desarrollado por los autores 


\section{Configuración cognitiva activada en la respuesta de Victor (Figura 4).}

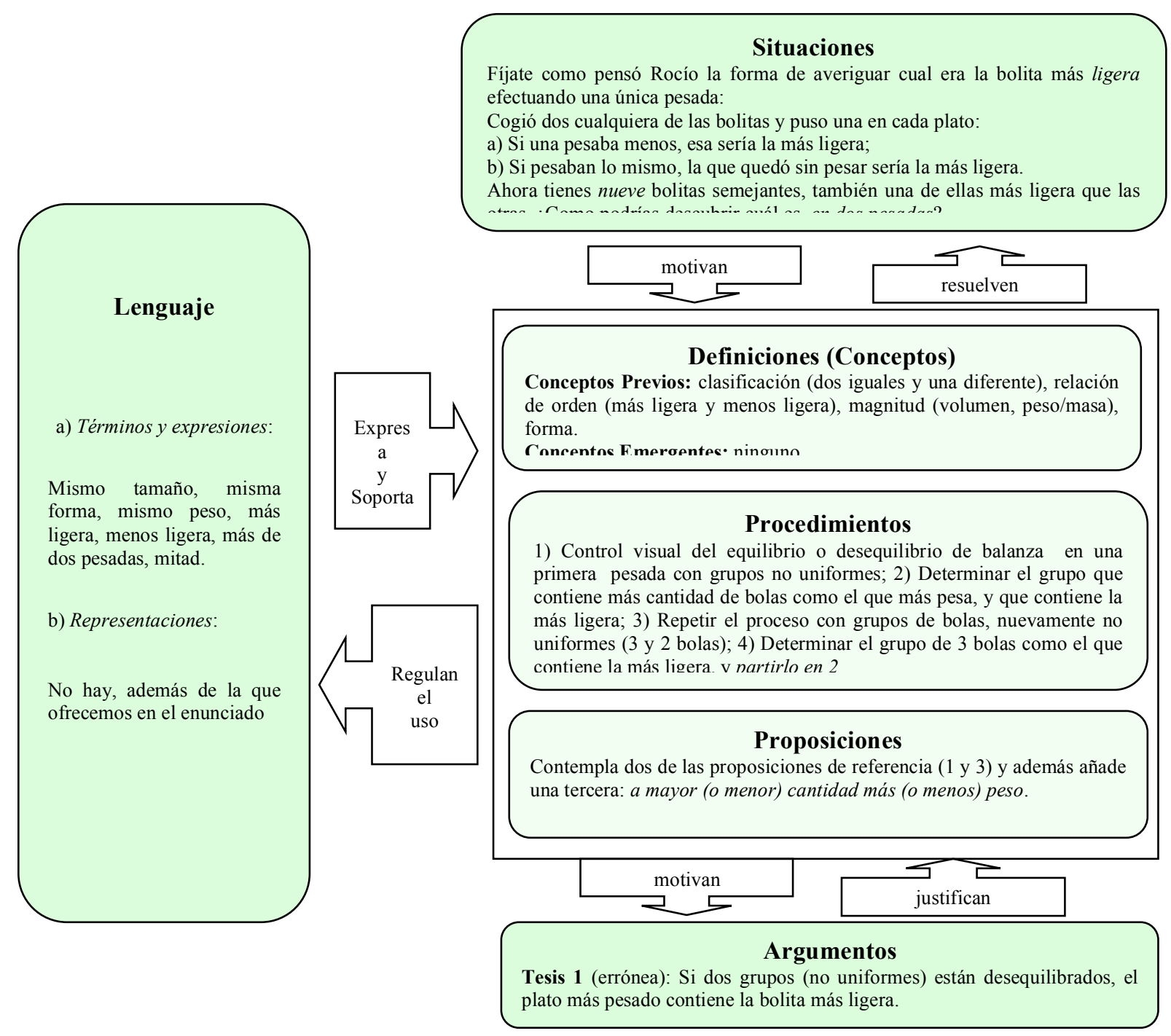

Figura 4 - Configuración cognitiva del protocolo escrito de Víctor Fuente: Desarrollado por los autores

De modo general y, en relación a la sucesión de acciones operativas y discursivas para la resolución de la tarea, podemos decir que Víctor al no percibir que se podría establecer el rango (peso relativo) de las bolitas sin necesidad de contrastarlas todas entre sí, sus acciones se centran en hacer comparaciones, primeramente con todas las bolas por bloques (no uniformes) después, tras la observación, elige el grupo que tiene más cantidad de bolas y vuelve a hacer comparaciones por bloques (no uniformes) hasta agotar los agrupamientos. Utiliza varias pesadas sin tener en cuenta las exigencias de la tarea. Reconoce el grupo que contiene más cantidad como el grupo que pesa más y como el que contiene la bola más ligera. Tiene dificultades para interpretar el grupo con menos cantidad de bolas.

A pesar de aplicar una estrategia incorrecta, las acciones discursivas de Víctor van en el sentido de justificar sus acciones mediante inferencias a partir de las posiciones de los 
platos de la balanza: si los dos grupos están desequilibrados, el plato más bajo contiene la bolita más ligera ( $\sin$ considerar que la posición de la bola más ligera no modifica la condición de mayor peso del grupo que tiene más cantidad de bolas). Sus justificaciones se basan en la propiedad de a más cantidad, más peso. Son argumentaciones limitadas, pero pensamos que reflejan el entorno (en el sentido de que no está solo, estando presentes, por ejemplo, los conceptos de masa y tamaño) de su objeto personal.

Configuración metacognitiva personal de Victor (Cuadro 2):

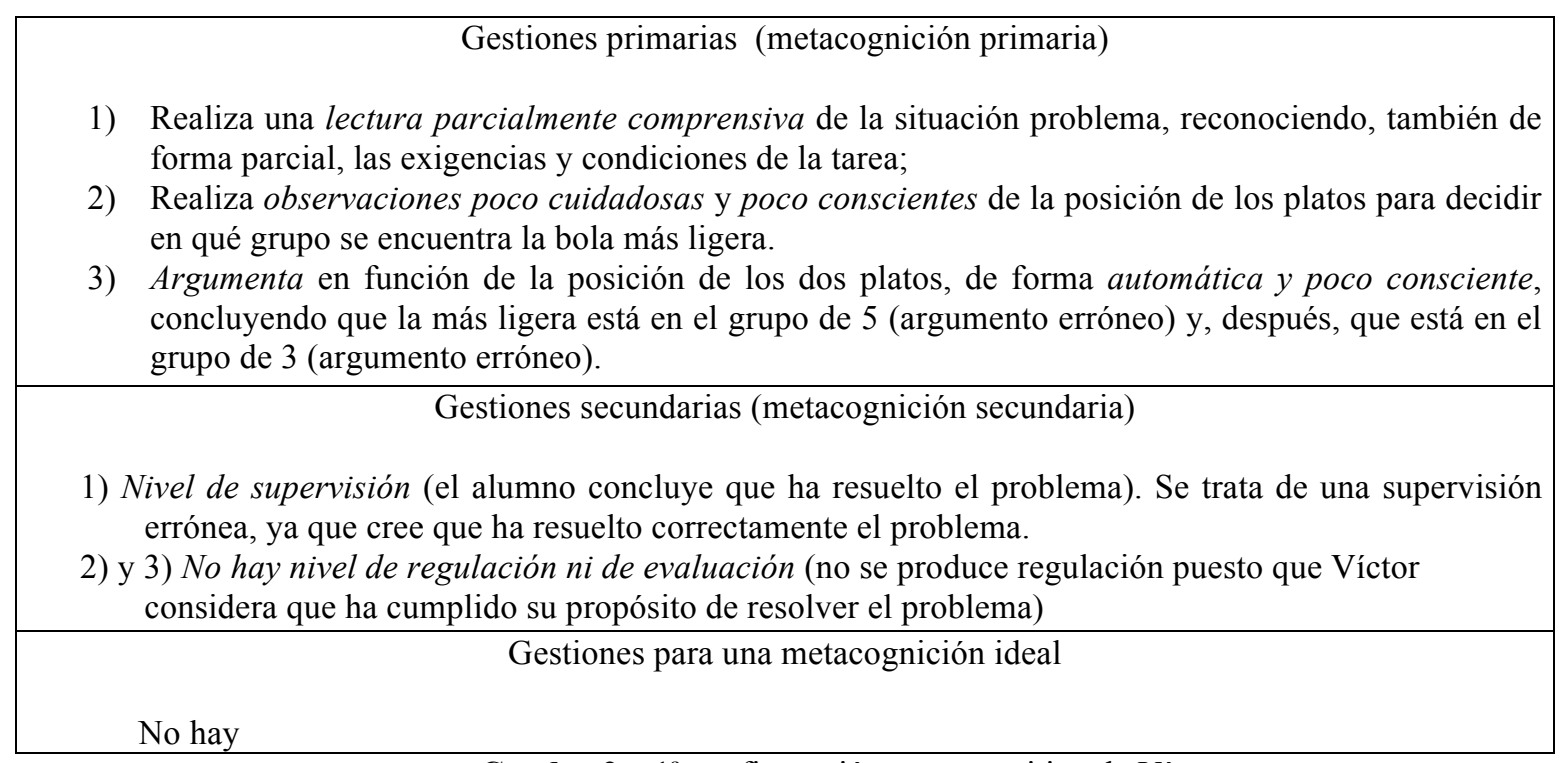

Cuadro $2-1^{\text {a }}$ configuración metacognitiva de Víctor Fuente: Desarrollado por los autores

De la misma forma no vamos a comentar cada elemento de esta otra configuración y remitimos a la tesis anteriormente mencionada.

Pero, también de modo general, observamos que sorprendentemente, Víctor había resuelto correctamente el problema de las 3 bolitas, siguiendo un proceso totalmente análogo al que se describe en el texto de las 9 bolitas. Todo ello nos llevó a considerar que el razonamiento por analogía no forma parte, todavía, de las competencias metacognitivas de Víctor.

Algunas conclusiones derivadas de este primer momento es que fue posible observar que el objeto personal masa, emergente de otras prácticas, es evocado aquí como fundamental para la realización de la práctica que Víctor describe y para la interpretación de los resultados. A partir de ese momento, sus acciones de comparar las bolas por bloques y de elegir el grupo que tiene más como el grupo donde está la bola más ligera se repiten. Sus acciones pasan por un nivel de experimentaciones selectivas en función del contexto que, por carencias de actividades de monitoreo (supervisión, regulación y evaluación) adecuados, no puede 
discriminar el grupo en donde se encuentra la bola más ligera y opta por elegir el que tiene más cantidad de bolas como el que pesa más y como el que contiene la bola más ligera. Estas carencias de acciones (o actividades) metacognitivas le llevan a proseguir con este criterio selectivo hasta el final del proceso.

Víctor no puede generalizar, ya que no percibe ninguna conexión con el problema anterior (de las 3 bolitas), lo que dificultó la aparición de estrategias alternativas. Esto le conduce a hacer uso de una acción de experimentación ingenua, no sujeta a las condiciones del problema (dos pesadas).

Desde el punto de vista global de las intenciones y propósitos, las acciones sucesivas de distribuir las bolas en grupos no uniformes tienen la intención de encontrar la bolita más ligera y, además, la intención de usar varias pesadas. Intenciones que son satisfechas. El propósito de resolver el problema (que consiste en encontrar la bolita más ligera), y que coincide con una de las intenciones, parece ser satisfactorio para Víctor, al no tener en cuenta las condiciones impuestas por la tarea. Si Víctor fuese consciente de estas condiciones su propósito no quedaría satisfecho, ya que optó por un método incorrecto de resolución. Quizás el nivel de satisfacción, consecuencia de la estrategia tomada (que para él era correcta) y el abandono de la condiciones del problema, le han impedido supervisar, evaluar y, por tanto, regular sus acciones tomando otro rumbo.

A la luz del análisis de este primer momento del caso Víctor bajo la óptica de las dos configuraciones aquí desarrolladas (cognitiva y metacognitiva) parece confirmar una de nuestras hipótesis (H1). El hecho de que una persona tenga adquiridos los conocimientos cognitivos suficientes para la realización exitosa de una práctica no siempre es garantía de éxito, y puede que no consiga resolverla, debido a carencias o uso incorrecto de conocimientos metacognitivos.

Para comprobar esta hipótesis realizamos una entrevista oral con Víctor, la cual caracteriza el segundo momento, dado a continuación. 


\section{Segundo momento: la entrevista (Cuadro 3).}

$\operatorname{VICTOR}(V):$ (momento de lectura)

1.V: Pones 4 en un lado y 5 en el otro y el lado que pesa más está la más ligera. Donde está la más ligera lo divide a la mitad y el lado que pese más, estará la más ligera.

2.ENTREVISTADORA(E): La mitad, ¿cómo?

3.V: Coge 3 y 2. Donde están las 3 y eso lo divide en 2 y ya está. 2 y 1.

4.E: Y si la más ligera estuviera en el plato de las 2 ?

5.V: Lo divides nuevamente.

6.E: Pero estarás usando más de dos pesadas, ¿no? [pausa].¿Crees que con esas dos pesadas descubrirás la bolita más ligera?

7.V: No.

8.E: ¿Puedes imaginar entonces otra forma de descubrir la más ligera?

9.V: No lo sé.

10.E: ¿Otra forma de agrupar? [larga pausa].

Volvamos, entonces, a lo que has dicho anteriormente, al principio. Agrupas en 4 y 5. Explícame eso despacito. 11.V: Con 5 y 4... como una es más ligera... va pesar más incluso... la que tenga así [hace gestos con las manos]... Espera. [pausa]... No se puede saber tampoco así... [larga pausa]... No se puede saber.

12.E: ¿No?

13.V: No, porque si la ligera está donde pones las de 4, va pesar menos pero aunque... aunque la ligera estuviese donde las 5, iba a ser lo mismo... [siempre pesará más el plato donde están las 5].

14. E: Y... que más?

15.V: No, no se puede.

\section{Cuadro 3 - La entrevista}

Fuente: Desarrollado por los autores

\section{Trayectoria Argumentativa (Cuadro 4):}

Tesis 1. Pones 4 en un lado y 5 en el otro y en el lado que pesa más está la más ligera. (episodio 1a (EP1a).

Tesis 2. Donde está la más ligera lo divide a la mitad y en el lado que pese más, estará la más ligera (EP1b).

Argumentos que invalidan la tesis 2: La mitad, ¿cómo? (EP2) ¿y si la más ligera estuviera en el plato de las 2? (EP4).

Argumentos que intentan avalar la tesis 2: Coge 3 y 2 [para dividir a la mitad]. (EP3a); donde están las 3, lo divide en 2 y ya está: 2 y l (EP3b); lo divides nuevamente (EP5)

Tesis 3. En dos pesadas [con este proceso] no se resuelve el problema (EP6).

Argumento que valida la tesis 3: No [reconocimiento de la validez de la tesis 3] (EP7).

Argumento que da cuenta de un bloqueo metacognitivo: No lo sé [como emprender otras estrategias] (EP9).

Argumento para intentar un desbloqueo: Agrupas en 4 y 5. Explícame eso despacito. (EP10)

Tesis 4: Con 5 y 4 [agrupando en] no se puede saber [donde está la más ligera] (EP11).

Argumentos: El plato donde están las 5 siempre pesará más (EP13); No, no se puede (EP15)

Cuadro 4 - Extracto de la entrevista (1)

Fuente: Desarrollado por los autores

Después de trazar la trayectoria argumentativa de Víctor pasamos a diseñar su segunda configuración metacognitiva, resultado de su entrevista.

Evolución de la configuración metacognitiva de Victor, como consecuencia de la entrevista (Cuadro 5): 


\section{Gestiones secundarias (metacognición secundaria)}

1) Nivel de supervisión: Reconocimiento consciente de que la estrategia elegida no permite decidir, en dos pesadas, dónde está la bola más ligera.

2) y 3) Nivel de regulación / evaluación. Consciencia de haber aportado una justificación errónea: el grupo "que pesa más" (identificado, correctamente, con el que tiene mayor cantidad de bolas) no tiene porqué contener la bola más ligera. Evidencia de no disponer, en ese momento, de una estrategia alternativa para planificar acciones que le permitan alcanzar la meta respetando las condiciones de la tarea.

Gestiones para una metacognición ideal

No hay (no percibe la aplicación de la analogía/transferencia del problema de las 3 bolitas al problema actual)

\section{Cuadro 5 - $2^{\mathrm{a}}$ configuración metacognitiva de Víctor \\ Fuente: Desarrollado por los autores}

Consideraciones sobre la evolución de la configuración metacognitiva de Víctor, tras la entrevista:

La primera pregunta de la entrevistadora tiene por objetivo una aclaración del método seguido por Víctor (La mitad, ¿cómo?).

La segunda pregunta de la entrevistadora tiene por objetivo hacerle ver a Víctor que no ha terminado de resolver el problema (¿Y si la más ligera estuviera en el plato de las 2?). Víctor se da cuenta de que no ha terminado el problema, es decir, que no ha satisfecho su propósito, y responde explicando cómo terminaría el problema (Lo divides nuevamente) con lo que ahora sí que considera que ha satisfecho el propósito de resolver el problema.

La tercera pregunta tiene por objetivo hacerle ver a Víctor que la manera en la que él cree haber resuelto el problema no cumple las condiciones del enunciado (Pero estarás usando más de dos pesadas, ¿no?... ¿Crees que con esas dos pesadas descubrirás la bolita más ligera?). Las preguntas dos y tres de la entrevistadora pretenden que Víctor realice acciones metacognitivas secundarias de supervisión. Este objetivo se logra, ya que Víctor se da cuenta de que no ha respetado las condiciones del enunciado (No).

La pregunta cuatro de la entrevistadora pretende que Víctor realice acciones metacognitivas secundarias de regulación sin ninguna ayuda externa, es decir que emprenda un nuevo camino para resolver el problema (¿Puedes imaginar entonces otra forma de descubrir la más ligera?). Este objetivo no se logra ya que Víctor no es capaz de realizar acciones metacognitivas ideales, o sea de darse cuenta de la analogía que hay entre este problema y el problema de las tres bolitas (No lo sé.)

La pregunta cinco de la entrevistadora pretende que Víctor realice acciones metacognitivas secundarias de regulación gracias a su ayuda, es decir que emprenda un nuevo camino para resolver el problema agrupando las bolitas de otra manera (¿Otra forma de 
agrupar?... Volvamos, entonces, a lo que has dicho anteriormente, al principio. Agrupas en 4 y 5. Explícame eso despacito.). Este objetivo se logra en parte, ya que en el resto del protocolo de Víctor se da cuenta de que su argumentación inicial de que la bolita más ligera estará en el grupo de 5 bolitas no es correcto (Cuadro 6): 11.V: Con 5 y 4 ... como una es más ligera... va pesar más incluso... la que tenga así (hace gestos con la mano)...
espera. (pausa)... no se puede saber tampoco así... (larga pausa)... no se puede saber.
12.E: ¿No?
13.V: No, porque si la ligera está donde pones las de 4, va pesar menos pero aunque... aunque la ligera
estuviese donde las 5 , iba a ser lo mismo...
14.E: Y... ¿qué más?
$15 . \mathrm{V}:$ No, no se puede...

\section{Cuadro 6 - Extracto de la entrevista (2) \\ Fuente: Desarrollado por los autores}

Víctor no es capaz, en este momento, de analizar estrategias nuevas para intentar resolver el problema. La evolución de sus competencias metacognitivas queda, por tanto, limitada a la consciencia de que su estrategia no es la idónea para resolver la tarea, sin poder aportar estrategias alternativas.

Como conclusiones derivadas de este según momento observamos que lo que resulta significativo es que, para darse cuenta de su error, Víctor utiliza las componentes de su configuración cognitiva de manera correcta (cuando reconoce que donde hay más bolas siempre pesa más, al margen de donde esté la bola más ligera). De modo que, en la entrevista, se confirma la hipótesis $H 1$, en el sentido de que, para este caso en especifico, la dificultad que tiene Víctor para resolver el problema propuesto se debe más a su configuración metacognitiva que a su configuración cognitiva: después de haber usado, previamente, una estrategia óptima para resolver el problema de las 3 bolitas, no es capaz de aplicar la analogía para reducir el caso de las 9 bolitas (agrupando uniformemente en grupos de 3) a pesar de ser (implícitamente) sugerida en el propio enunciado del problema.

\section{Consideraciones finales}

Del análisis de los resultados del caso Víctor fue posible confirmar la hipótesis formulada. Para o caso en particular, el error de Víctor parece que no se debe a fallos en su configuración cognitiva, puesto que sólo tiene dificultades con el componente argumentativo de la configuración epistémica. Su respuesta errónea parece que se debe, básicamente, a su configuración metacognitiva.

De modo general, y a partir de los resultados de la PHM (que si muestran en Gusmão (2006)), se concluye que una vez más la relación de dependencia entre estas dos competencias 
así como el mutuo apoyo que puede haber entre ambas de cara a la comprensión de las situaciones problemas y de sus procesos de resolución.

Considerase que las competencias cognitivas y metacognitivas de los resolutores vienen manifestadas en armonía. Cuando los resolutores presentan carencias significativas en su configuración cognitiva, también presentan paralelamente carencias en sus configuraciones metacognitivas. A medida que utilizan configuraciones cognitivas más elaboradas, las configuraciones metacognitivas se muestran como un catalizador para la evolución de las primeras, aproximándolas paulatinamente a la configuración epistémica de referencia. Recíprocamente, el poseer una configuración cognitiva desarrollada hace paralelamente aflorar una configuración metacognitiva que, a su vez, se aproxima a la configuración metacognitiva de referencia.

\section{Referencias}

CAJ, J. A protocol-analytic study of metacognition in mathematical problem-solving. Mathematics Educational Research Journal, Australia, v. 1-2, n. 6, p. 66-183. Dec. 1994.

CARREL, P.; GAJDUSEK, L.; WISE, T. Metacognition and EFL/ESL reading. In: HARTMAN, H. J. (Ed). Metacognition in learning and instruction: theory, research and practice. New York: Kluwer Academic Publishers, 2001. p. 229-243.

CHEVALLARD, Y. Concepts fondamentaux de la didactique: perspectives apportées par une approche anthropologique. Recherches en Didactique des Mathématiques, Grenoble, v. 12, n. 1, p. 73-112, 1992.

CLARKE, D. J.; STEPHENS, W. M.; WAYWOOD, A. Communication and the learning of mathematics. In: ROMBERG, T. A. (Ed.). Mathematics assessment and evaluation: imperatives for mathematics educators. New York: The State University of New York Press, 1992. p. 184-212.

FERNANDES, D. Aspectos metacognitivos na resolução de problemas de matemática. Educação e Matemática. Revista Educação \& Matemática da Associação de Professores de Matemática, Lisboa, v. 4, n. 8, p. 3-6, nov./dez. 1988.

FLAVELL, J. H. Metacognitive aspects of problem solving. In: RESNICK, L. (Ed.). The nature of intelligence. Hillsdale, NJ: Lawrence Erlbaum Associates, 1976. p. 231-235.

FLAVELL, J. H. Monitoring social cognitive enterprises: something else that may develop in the area of social cognition. In: FLAVELL, J. H.; ROSS, L. (Ed.). Social cognitive development: frontiers abd possible futures. New York: Cambridge university Press, 1981. p. 272-287.

FLAVELL, J. H. Speculation about the motive and development of metacognition. In: WEINERT, F.; KLOWE, R. (Ed.). Metacognition, Motivation and Understanding. Hillsdale, New Jersey: Lawrence Erlbaum Associates, Publishers, 1987. p. 21-29.

FLAVELL, J. H.; WELLMAN, H. Metamemory. In: KAIL JR, R. V.; HAGEN, J. W. (Ed.). Perspectives on the development of memory and cognition. Hillsdale, NJ: Lawrence Erlbaum Associates, 1977. p. 3-33. 
FONT, V.; GODINO, J. D. La noción de configuración epistémica como herramienta de análisis de textos matemáticos: su uso en la formación de profesores. Educação Matemática Pesquisa, São Paulo, v. 8, n. 1, p. 67-98. 2006.

FONT, V.; PLANAS, N.; GODINO, J. D. Modelo para el análisis didáctico en educación matemática. Infancia y Aprendizaje, Madri, ES, v. 33, n. 1, p. 89-105, 2010.

GAROFALO, J.; LESTER, F. K. Metacognition, cognitive monitoring, and mathematical performance. Journal for Research in Mathematics Education, Reston, Virginia, v. 16, n. 3, 163176. May 1985.

GODINO, J. D.; BATANERO, C. Significado institucional y personal de los objetos matemáticos. Recherches en Didactique des Mathématiques, Grenoble, Franca, FR, v. 14, n. 3, p. 325-355, 1994.

GODINO, J. D.; BATANERO, C.; FONT, V. The onto-semiotic approach to research in mathematics education. ZDM. The International Journal on Mathematics Education. Turkey, v. 39, n. 1-2, p. 127-135, 2007.

GONÇALVES, M. C. M. A influência da Metacognição na aprendizagem: uma intervenção realizada na aula de matemática. 1996. Dissertação (Mestrado) Faculdade de Ciências Humanas. Universidade Católica Portuguesa. Lisboa, Portugal, 1996.

GONZÁLEZ, F. Procesos cognitivos y metacognitivos que activan los estudiantes universitarios venezolanos cuando resuelven problemas matemáticos. 1997. 551 f. Tesis (Doctoral en Educación) - Universidad de Carabobo, Carabobo, Venezuela, 1997.

GOOS, M. Metacognitive decision making and social interactions during paired problem solving. Mathematics Education Research Journal, Reston, Virginia, v. 6, n. 2, p. 144-165, Dec. 1994.

GUSMÃO, T. C. R. S. Los procesos metacognitivos en la comprensión de las prácticas de los estudiantes cuando resuelven problemas matemáticos: una perspectiva ontosemiótica. $2006.366 \mathrm{f}$. Tesis (Doctorado en Didáctica de las Matemáticas) - Universidade de Santiago de Compostela, Santiago de Compostela, España, 2006.

GUSMÃO, T. C. R. S.; FONT, V.; CAJARAVILLE, J. A. Análises cognitivo e metacognitivo de práticas matemáticas de resolução de problemas: o caso Nerea. Educação Matemática Pesquisa, São Paulo, v. 11, n. 1, p. 8-43, 2009.

KAUNE, C. Reflection and metacognition in mathematics education - tools for the improvement of teaching quality. ZDM-The International Journal on Mathematics Education, Turkey, v. 38, n. 4, p. 350-360, Jan. 2006.

LESH, R.; ZAWOJEWSKI, J. S. Problem solving and modeling. In: LESTER, F. K. (Ed.). The Second Handbook of Research on Mathematics Teaching and Learning. National Council of Teachers of Mathematics. Charlotte, NC: Information Age Publishing, 2007. p. 763-804.

LESTER, F. K. Musings about mathematical problem-solving research: 1970-1994. Journal for Research in Mathematics Education, Reston, US, v. 25, n. 6, p. 660-675, 1994.

LESTER, F. K.; GAROFALO, J. Mathematical problem solving: issues in research. Philadelphia, PA: Franklin Institute Press, 1982.

LUDKE, M.; ANDRÉ, M. E. A Pesquisa em Educação: abordagens qualitativas. São Paulo: EPU, 1986. 
MATEOS, M. Metacognición y educación. Buenos Aires, Argentina: Aique Grupo Editor, 2001. Serie Psicología Cognitiva y Educación.

NCTM. Principles and standards for school mathematics. Reston VA. USA. (Traducción castellana: Sociedad Andaluza de Educación Matemática THALES (2003). Principios y Estándares para la Educación Matemática. Granada), 2003.

OCDE. The PISA 2003 Assessment Framework: Mathematics, Reading, Science and Problem Solving Knowledge and Skills. París, OCDE, 2003.

RODRÍGUEZ, E. Metacognición, matemáticas y resolución de problemas: una propuesta integradora desde el enfoque antropológico. 2005. $904 \mathrm{f}$. Tesis (Doctorado en Didáctica de las Matemáticas) - Universidad Complutense de Madrid, Madrid, 2005.

RODRÍGUEZ, E.; BOSCH, M.; GASCÓN, A. A networking method to compare theories: metacognition in problem solving reformulated within the Anthropological Theory of the Didactic. ZDM - The International Journal on Mathematics Education, Turkey, v. 40, n. 2, p. 287-301, Apr. 2008.

SCHOENFELD, A. H. Mathematical problem solving. London, United Kingdom: Academic Press Inc. (London) Ltd, 1985.

SCHOENFELD, A. H. Learning to think mathematically: Problem solving, metacognition, and sense making in mathematics. In: GROWS, D. A. (Ed.). Handbook of Research on Mathematics Teaching and Learning, New York: Macmillan, 1992. p. 334-370.

SCHOENFELD, A. H. Problem solving in the United Status, 1970-2008: research and theory, practice and politics. ZDM - The International Journal on Mathematics Education, Turkey, v. 39, n. 5-6, p. 537-551, Sept. 2007.

SCHRAW, G. Promoting general metacognitive awareness. In: HARTMAN, H. J. (Ed.).

Metacognition in learning and instruction: theory, research and practice. New York, EUA: Kluwer Academic Publishers, 2001. p. 3-16.

SILVER, E. A.; MARSHALL, S. P. Mathematical and scientific problem solving: findings, issues, and instructional implications. In: DINS, B. F.; JONES, L. (Ed.). Dimensions of thinking and cognitive instruction. Hillsdale, NJ: Erlbaum, 1990. p. 265-290.

YUSSEN, S. The role of metacognition in contemporary theories of cognitive development. In: FORREST-PRESSLEY, L.; MACKINNON, G. E. M.; WALLER, G. (Ed.). Metacognition, cognition, and human performance. Theoretical Perspectives. London: Academia Press, Inc., 1985. p. 253-283. 